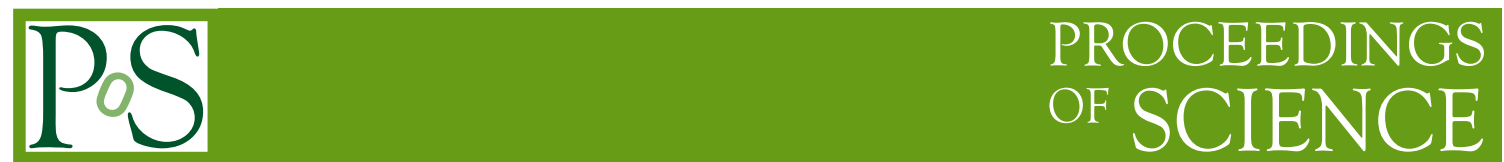

\title{
Gluino pair production at threshold
}

\section{Peter Marquard*}

TTP, Karlsruhe Institute for Technology (KIT)

E-mail: peter.marquardekit.edu

We present a next-to-leading order calculation for the production of gluino pairs near threshold at the LHC. We show that threshold effects lead to a characteristic shape of the differential cross section around threshold and an increase of the total cross section.

36th International Conference on High Energy Physics

4-11 July 2012

Melbourne, Australia

*Speaker. 


\section{Introduction}

The search for new particles, predicted in supersymmetric models, is one of the important tasks of the experiments at the Large Hadron Collider (LHC). The detailed determination of particle masses and couplings will be crucial for the discrimination between various manifestations of supersymmetry (SUSY) and alternative models, even more so if one wants to distinguish between the different variants of supersymmetric models, to identify the origin of breaking of supersymmetry and to measure the model parameters. One of the SUSY signals will be events with missing energy or missing transverse momentum, resulting from cascade decays of squarks and gluinos into the lightest supersymmetric particle (LSP) which escapes detection. The existence of squarks, gluinos and the LSP is definitely a key prediction of supersymmetry.

The importance of squark and gluino searches has motivated a series of detailed studies of hadroproduction cross sections for squarks and gluinos. The lowest order has been evaluated long time ago $[1,2,3]$. Subsequently the next-to-leading order (NLO) SUSY-QCD corrections were calculated $[4,5,6]$, more recently the effect of soft-gluon resummation $[7,8,9,10,11,12,13]$ was included. The present paper will be concerned with gluino-pair production close to threshold, which exhibits a number of peculiar features.

As a consequence of their colour-octet representation the production cross section of gluinos is large and perturbative corrections are particularly important. Furthermore, the threshold region is strongly affected by final state interaction, which in leading order is related to Sommerfeld rescattering corrections and which, compared to the similar situation in top-anti-top production [14], is amplified by the ratio $\left(C_{A} / C_{F}\right)^{2}=(9 / 4)^{2}$.

\section{Calculation and Results}

The differential cross section with respect to the invariant mass of the gluino pair can be obtained by integrating the partonic cross section of a given colour configuration $T$ over the luminosity function

$$
M \frac{\mathrm{d} \sigma_{P P \rightarrow T}}{\mathrm{~d} M}\left(S, M^{2}\right)=\sum_{i, j} \int_{\rho}^{1} \mathrm{~d} \tau\left[\frac{\mathrm{d} \mathscr{L}_{i j}}{\mathrm{~d} \tau}\right]\left(\tau, \mu_{F}^{2}\right) M \frac{\mathrm{d} \hat{\sigma}_{i j \rightarrow T}}{\mathrm{~d} M}\left(\hat{s}, M^{2}, \mu_{R}^{2}, \mu_{F}^{2}\right)
$$

with

$$
\begin{gathered}
M \frac{\mathrm{d} \hat{\sigma}_{i j \rightarrow T}}{\mathrm{~d} M}\left(\hat{s}, M^{2}, \mu_{R}^{2}, \mu_{F}^{2}\right)=\mathscr{F}_{i j \rightarrow T}\left(\hat{s}, M^{2}, \mu_{R}^{2}, \mu_{F}^{2}\right) \frac{1}{m_{\tilde{g}}^{2}} \operatorname{Im}\left\{G^{[1,8,10,27]}\left(0, M-2 m_{\tilde{g}}+i \Gamma_{\tilde{g}}\right)\right\}, \\
{\left[\frac{\mathrm{d} \mathscr{L}_{i j}}{\mathrm{~d} \tau}\right]\left(\tau, \mu_{F}^{2}\right)=\int_{0}^{1} \mathrm{~d} x_{1} \int_{0}^{1} \mathrm{~d} x_{2} f_{i \mid P}\left(x_{1}, \mu_{F}^{2}\right) f_{j \mid P}\left(x_{2}, \mu_{F}^{2}\right) \delta\left(\tau-x_{1} x_{2}\right),}
\end{gathered}
$$

where $\mathscr{F}_{i j \rightarrow T}\left(\hat{s}, M^{2}, \mu_{R}^{2}, \mu_{F}^{2}\right)$ denotes the hard scattering kernel. The shape of the cross section near threshold is therefore governed by the shape of the Green's function for the corresponding color state, which is shown in Fig. 1. The Green's function can be obtained from the result for the top-quark system [16]

$$
G^{[R]}\left(0, M-2 m_{\tilde{g}}+i \Gamma_{\tilde{g}}\right)=i \frac{v m_{\tilde{g}}^{2}}{4 \pi}+\frac{C^{[R]} \alpha_{s}\left(\mu_{G}\right) m_{\tilde{g}}^{2}}{4 \pi}\left[g_{\mathrm{LO}}+\frac{\alpha_{s}\left(\mu_{G}\right)}{4 \pi} g_{\mathrm{NLO}}+\ldots\right],
$$




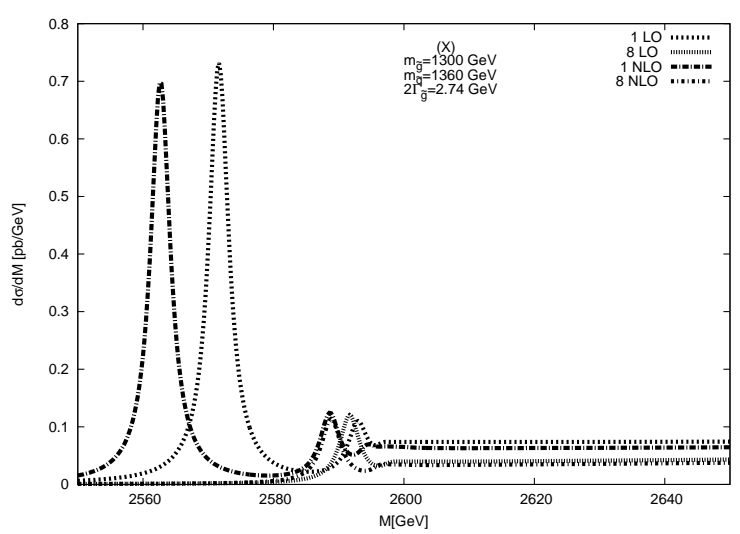

Figure 1: Green's functions for singlett and octett configuration at leading and next-to-leading order.

\begin{tabular}{c||c|c|c|c|c|c|}
$\begin{array}{c}\text { benchmark } \\
\text { point }\end{array}$ & $m_{\tilde{g}}[\mathrm{GeV}]$ & $\bar{m}_{\tilde{q}}[\mathrm{GeV}]$ & $\Delta M[\mathrm{GeV}]$ & $2 \Gamma_{\tilde{g}}[\mathrm{GeV}]$ & $\Gamma_{g g}[\mathrm{GeV}]$ & $\begin{array}{c}\text { dominant decay } \\
\text { channels }\end{array}$ \\
\hline \hline$(\mathrm{p})$ & 734.11 & 714.46 & 16.02 & 3.48 & 0.41 & $\tilde{g} \rightarrow \tilde{b}_{1} \bar{b}, \tilde{b}_{1}^{*} b$ \\
\hline$(\mathrm{X})$ & 1300.00 & 1360.00 & 26.21 & 3.66 & 1.23 & $\tilde{g} \rightarrow \tilde{t}_{1} \tilde{t}, \tilde{t}_{1}^{*} t$ \\
\hline$(\mathrm{Y})$ & 1370.00 & 1235.00 & 27.27 & 20.00 & 1.26 & $\tilde{g} \rightarrow \tilde{t}_{1} \tilde{t}, \tilde{t}_{1}^{*} t$
\end{tabular}

Table 1: Comparison of gluino masses, average squark masses, gluinonium level spacing, single decay rate, annihilation rate and dominant decay channels for the benchmark points under investigation.

with

$$
\begin{gathered}
g_{\mathrm{LO}} \equiv L-\psi^{(0)} \\
g_{\mathrm{NLO}} \equiv \beta_{0}\left[L^{2}-2 L\left(\psi^{(0)}-\kappa \psi^{(1)}\right)+\kappa \psi^{(2)}+\left(\psi^{(0)}\right)^{2}-3 \psi^{(1)}-2 \kappa \psi^{(0)} \psi^{(1)}\right. \\
\left.\quad+4{ }_{4} F_{3}(1,1,1,1 ; 2,2,1-\kappa ; 1)\right]+a_{1}\left[L-\psi^{(0)}+\kappa \psi^{(1)}\right],
\end{gathered}
$$

and

$$
\kappa \equiv i \frac{C^{[R]} \alpha_{s}\left(\mu_{G}\right)}{2 v}, \quad v \equiv \sqrt{\frac{M-2 m_{\tilde{g}}+i \Gamma_{\tilde{g}}}{m_{\tilde{g}}}}, \quad L \equiv \ln \frac{i \mu_{G}}{2 m_{\tilde{g}} v} .
$$

The $n$-th derivative $\psi^{(n)}=\psi^{(n)}(1-\kappa)$ of the digamma function $\psi(z)=\gamma_{E}+(d / d z) \ln \Gamma(z)$ is evaluated at $(1-\kappa)$ and ${ }_{4} F_{3}$ denotes the Hypergeometric function.

The hard scattering kernel has to be calculated for the different colour states by applying suitable colour projectors and contains both virtual and real corrections. The real corrections have to be taking into account since the virtual corrections exhibit infrared singularites which cancel when combined with the real corrections. The remaining singularities from initial-state radiation can be absorbed into the parton distribution functions. For the numerical evaluation we used the MSTW2008NLO [17] PDF set.

We have investigated three typical scenarios for squark and gluino masses which are shown in Tab. 1 in detail. In Figs. 2-4 we show the results for the cross section in the threshold region 


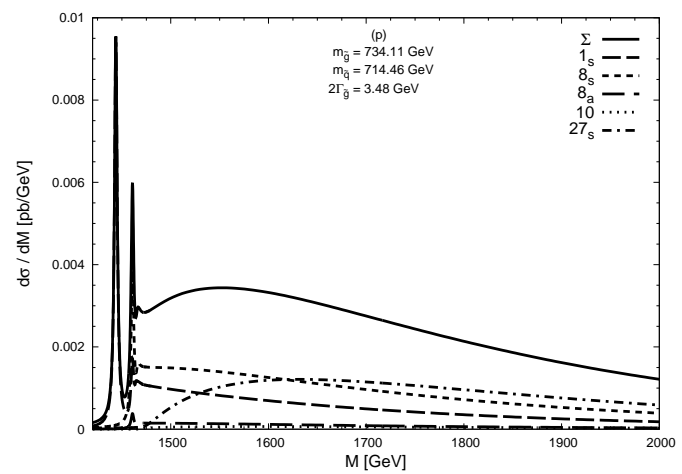

(a)

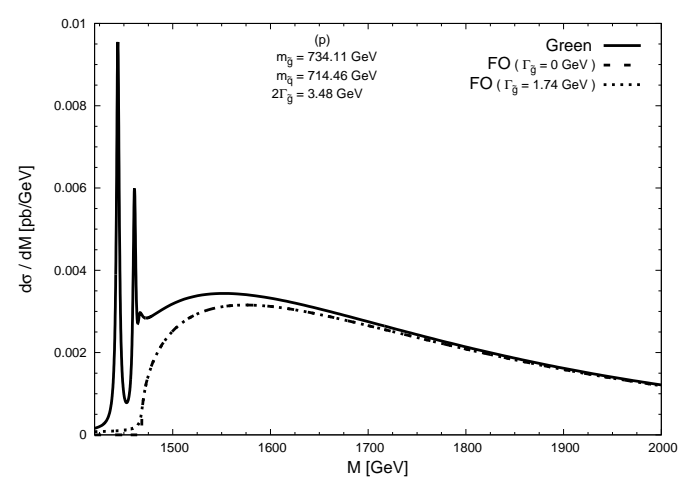

(c)

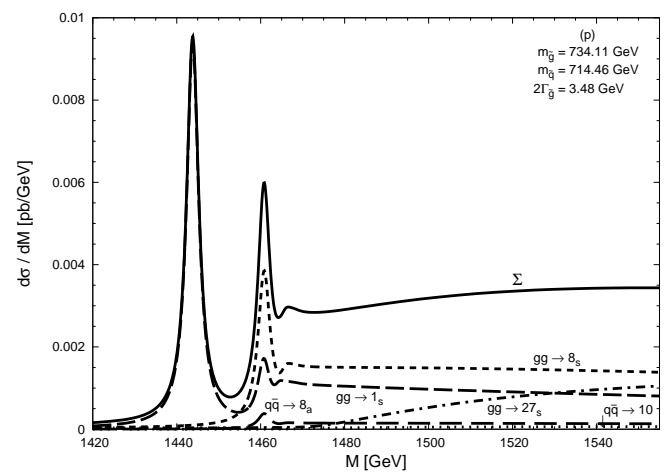

(b)

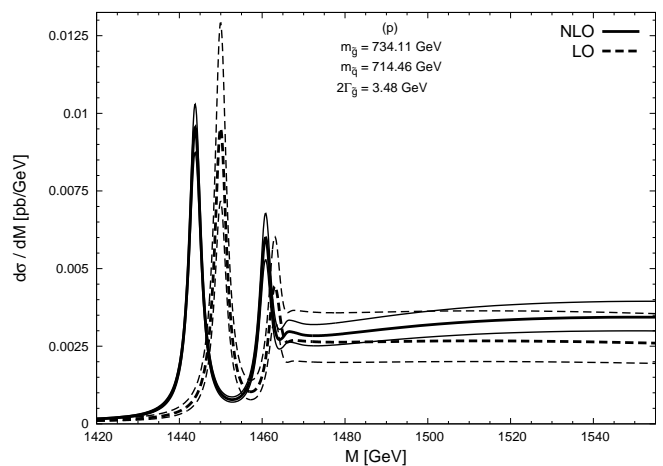

(d)

Figure 2: Cross section for light gluinos.

for these scenarios. For scenario (p) we show in Fig. 2 also the renormalization-scale dependence. As can be seen the scale dependence is reduced and in particular, the uncertainty at the position of the 1S-resonance is greatly improved. In Figs. 2(c)- 4(c) we show in addition the results for the differential cross using a fixed-order approach for the Green's function, i.e. replacing it by its expansion in $\alpha_{s}$ first keeping $\Gamma_{\tilde{g}}$ non-vanishing and then in the limit $\Gamma_{\tilde{g}} \rightarrow 0$

$$
\operatorname{Im} G^{[R]} \rightarrow \frac{m_{\tilde{g}}^{2}}{4 \pi} v\left(1+C^{[R]} \frac{\alpha_{s} \pi}{2 v}\right) .
$$

In this limit the result is an approximation of the fixed-order calculation. The difference can be understood as the threshold enhancement missing in the fixed-order calculation. Comparing the integrated total cross sections the enhancement amounts to typically $7 \%$ to $9 \%$.

\section{Conclusions}

The next-to-leading order analysis for hadronic production of gluino pairs close to threshold has been presented. The matching coefficients were evaluated separately for the different colour configurations of gluino pairs with relative angular momentum zero. The cross section is strongly affected by final-state interaction which is encoded in the NLO Green's function and which depends on the gluino decay rate, the colour configuration and the invariant mass of the pair. We have investigated three different SUSY-scenarios covering a wide range of gluino and squark masses, 


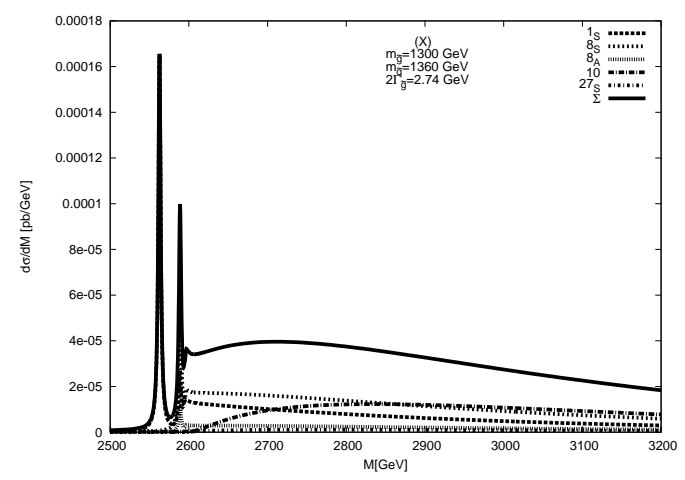

(a)

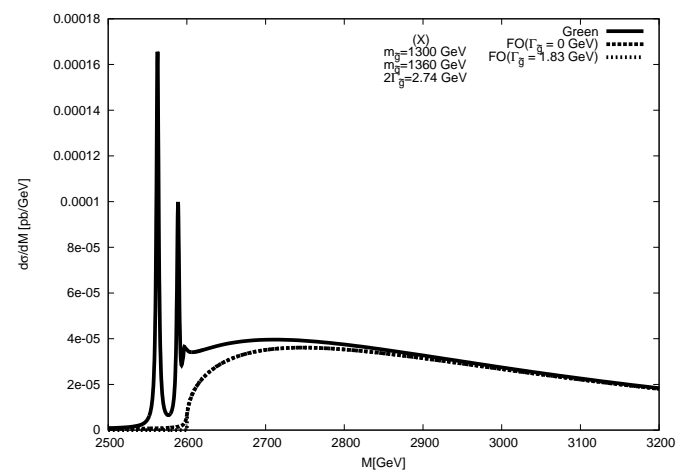

(c)

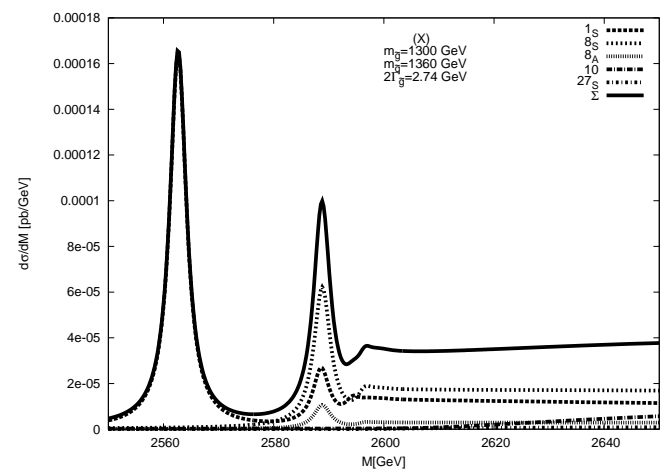

(b)

Figure 3: Cross section for heavy gluinos and a small decay width.

and studied the renormalization and factorization-scale dependence of the result. Compared to the leading-order prediction the result is more stable, and, for $\mu$ between $m_{\tilde{g}}$ and $4 m_{\tilde{g}}$, varies by $\pm 15 \%$. The complete NLO threshold production of $(\tilde{g} \tilde{g})$ boundstates considered in this paper enhances the fixed-order prediction by typically $7 \%$ to $9 \%$.

\section{Acknowledgements}

We like to thank M. Kauth, J.H. Kühn and M. Steinhauser for collaboration and T. Kasprzik for reading of the manuscript.

\section{References}

[1] P. R. Harrison and C. H. Llewellyn Smith, Nucl. Phys. B 213 (1983) 223 [Erratum-ibid. B 223 (1983) 542].

[2] H. E. Haber and G. L. Kane, Phys. Rept. 117 (1985) 75.

[3] S. Dawson, E. Eichten and C. Quigg, Phys. Rev. D 31 (1985) 1581.

[4] W. Beenakker, R. Hopker, M. Spira and P. M. Zerwas, Nucl. Phys. B 492 (1997) 51 [hep-ph/9610490].

[5] W. Beenakker, R. Hopker and M. Spira, hep-ph/9611232. 


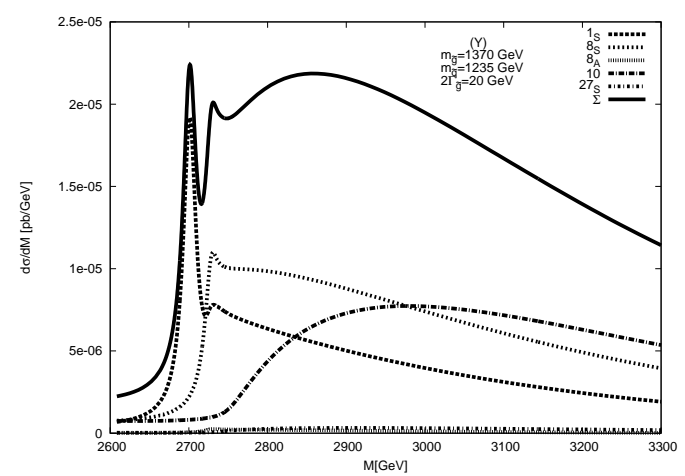

(a)

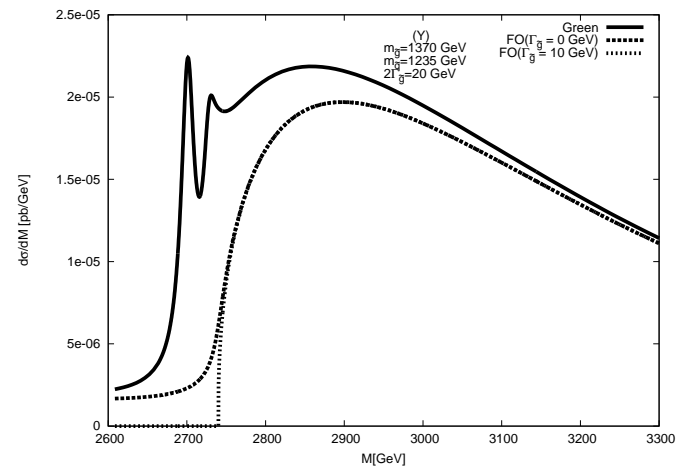

(c)

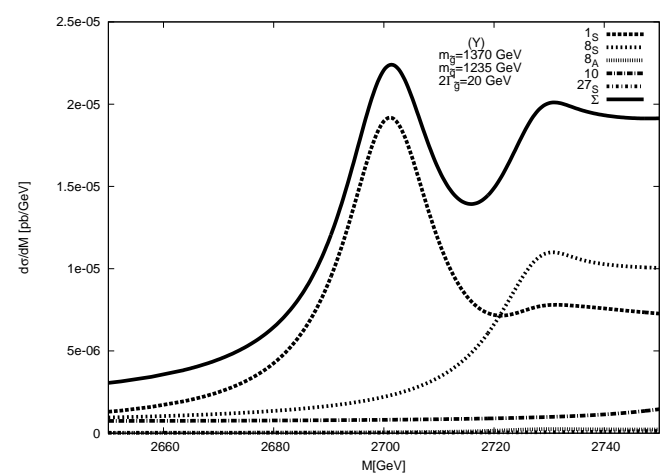

(b)

Figure 4: Cross section for heavy gluinos and a large decay width.

[6] W. Beenakker, M. Kramer, T. Plehn, M. Spira and P. M. Zerwas, Nucl. Phys. B 515 (1998) 3 [hep-ph/9710451].

[7] A. Kulesza and L. Motyka, Phys. Rev. Lett. 102 (2009) 111802 [arXiv:0807.2405 [hep-ph]].

[8] U. Langenfeld and S. -O. Moch, Phys. Lett. B 675 (2009) 210 [arXiv:0901.0802 [hep-ph]].

[9] A. Kulesza and L. Motyka, Phys. Rev. D 80 (2009) 095004 [arXiv:0905.4749 [hep-ph]].

[10] W. Beenakker, S. Brensing, M. Kramer, A. Kulesza, E. Laenen and I. Niessen, JHEP 0912 (2009) 041 [arXiv:0909.4418 [hep-ph]].

[11] W. Beenakker, S. Brensing, M. Kramer, A. Kulesza, E. Laenen and I. Niessen, JHEP 1008 (2010) 098 [arXiv:1006.4771 [hep-ph]].

[12] M. Beneke, P. Falgari and C. Schwinn, Nucl. Phys. B 842 (2011) 414 [arXiv:1007.5414 [hep-ph]].

[13] W. Beenakker, S. Brensing, M. Kramer, A. Kulesza, E. Laenen, L. Motyka and I. Niessen, Int. J. Mod. Phys. A 26 (2011) 2637 [arXiv:1105.1110 [hep-ph]].

[14] J. H. Kuhn and E. Mirkes, Phys. Rev. D 48 (1993) 179 [hep-ph/9301204].

[15] M. R. Kauth, J. H. Kühn, P. Marquard and M. Steinhauser, Nucl. Phys. B 857 (2012) 28 [arXiv:1108.0361 [hep-ph]].

[16] M. Beneke, A. Signer and V. A. Smirnov, Phys. Lett. B 454 (1999) 137 [hep-ph/9903260].

[17] A. D. Martin, W. J. Stirling, R. S. Thorne and G. Watt, arXiv:0901.0002 [hep-ph]. 